\title{
On a vertex-edge marking game on graphs
}

\author{
REVISION NOTES \\ Boštjan Brešar, Nicolas Gastineau, Tanja Gologranc, and Olivier Togni
}

Many thanks to the reviewers for their careful reading of the paper and for their remarks and suggestions that enabled us to improve the quality and the clarity of the paper. Following the comments of Reviewer \# 1, we have made some shortenings of the paper. In particular, we have removed some parts of the paper, mainly in Section 2, which had some issues and which have no effect on the rest of the paper containing main results. Following the comments of Reviewer \# 2, we have modified the main definitions so that they work for infinite graphs, and, whenever possible, the results were extended to arbitrary (also disconnected) graphs with multiple edges.

In the rest of the revision notes, we give more detailed answers to the main points of each of the reviewers. In addition, the corrections made are highlighted in the version of the paper attached to this revision note.

\section{Comments by Reviewer \# 1}

- Issue 1: I think there is an error at page 4, Line -4 and Line -3: "The degeneracy $\Delta^{+}(G)$ of a graph $G$ is the smallest $k$ such that $G$ is $k$-degenerate and is given by $\Delta^{+}(G)=2 d^{*}(G)=\operatorname{mad}(G)($ see $[14]) . "$

I think somewhere of the above equalities is not right ... Following above comments, the proof and contents of Theorem 4 of this paper is wrong.

The reviewer has a point. Anyway, taking into account some comments of both reviewers, we decided to remove this part of the paper in the revised version. In particular, we have removed the observations about several concepts, such as maximum density $d^{*}(G)$, maximum average degree $\operatorname{mad}(G)$, pseudoarboricity $p(G)$. There are several reasons why. Firstly, some of these concepts were defined just for finite graphs, but now we focus on general graphs. Secondly, we included these concepts in the paper just to present some upper bounds for $\operatorname{col}_{v e}(G)$ as functions of these parameters, but these concepts were not needed elsewhere. In addition, as noted by Reviewer \#1, most of these bounds are almost trivial and follow directly from the definitions, so we agree that they are not necessary.

- Issue 2: I think all the results and proofs of this paper are simple, but the presentations are unnecessarily long. I gave some concrete examples: Example 1: Corollaries 5, 6, and 7 should not be there at all.

We have removed them.

Example 2: All the results in Proposition 8 are very obvious, they can be done in a few sentences instead of using almost one page.

Done. This part has been considerably shortened. 
Example 3: For Section 4 "infinite lattice": this paper has nothing to do with infinite, the infinite there is only used in examples for some lower bounds, and the examples really does not need infinite (maybe a few hundreds (thousands?) vertices will be more than enough).

The reviewer is right. Following the suggestions of Reviewer \#2 we have modified the definition of the vertex-edge coloring game, so that it works for infinite graphs too. In addition, we have merged two sections (about planar graph and about infinite lattices) to one section entitled Planar graphs, making the material a bit more compact. After all, also the mentioned lattices are planar graphs.

Example 4: For Section 5 "Complete graphs": The proof for the main results is actually by using a kind of "greedy" strategy by Alice, is simple. But currently is three pages long, and presented in a somewhat abstract way; and this abstract way has done nothing more than just giving a long and complicate proof of some simple arguments; which I think is unnecessary. A direct proof (probably by induction) will give a clear presentation.

Initially, we were thinking that the result could be proved by a direct and short proof, but could not find one. We also think that the current proof is not too abstract, since, as you correctly notice, it only follows a kind of a greedy strategy for Alice. Even if the proof would not use the terminology of sorted words, we would essentially need some concept, which would emulate the idea of sorted words.

We also wish to emphasize, as we now do in the abstract, the introduction and in the formulation of Theorem 16, that the result holds not only for complete graphs $K_{n}$, but for any multigraphs whose underlying graph is $K_{n}$, which makes the result slightly stronger.

- Issue 3: The references have some problems. First example is as explained in Issue 1: the authors made some mistakes, but they say the results come from Reference [14]; and they don't say where; and indeed, according what I have checked, this is not the case. Second example: the authors uses a lot on the maximum out degree of orientations of $G$, and its relation with $\operatorname{mad}(G)$, this is Hakimi's well-known result. But there are no reference of Hakimi's paper; instead, the reference is kept on going to Reference [6]. Similarly, the paper mentions arboricity of graphs, all they used is not more than Nash-Williams and Tutte's well-known forests decomposition theorem. But Nash-Williams and Tutte's theorem is never mentioned, and again the reference is kept on going to Ref- erence [6].

Since the results dealing with density were only applicable to finite graphs and were not used in the rest of the paper, we have chosen to remove them.

\section{Minor comments}

All other comments of the reviewer have been taken into account and typos have been corrected. 


\section{Comments by Reviewer \# 2}

- (Comment 1) A natural definition of the score would be the following. The score of an unmarked vertex $\mathrm{v}$ at a certain state $t$ of the game is the number of marked edges incident with $\mathrm{v}$; the score of the vertex $\mathrm{v}$ is the supremum of the scores of $\mathrm{v}$ at state $t$, where the supremum is taken over all states $t$ of the game. In this paper, the score of a vertex $\mathrm{v}$ is defined as the number of marked edges incident with $\mathrm{v}$ at the time Alice marks the vertex v. These two concepts are the same for finite graphs but may be different in case of locally finite infinite multigraphs. According to your definition, the score of vertices that are never marked is undefined. Assuming that it is defined to be zero, then e.g. the final score might be zero for multigraphs with arbitrary maximum degree but infinitely many isolated vertices, which shows that this definition makes no sense at least in disconnected infinite multigraphs. By introducing an additional dominating vertex, the same construction applies to connected infinite multigraphs, resulting in a final score of one. Although you restrict your considerations to connected multigraphs, using the above given more natural definition might be what you might have wanted to define, and the more natural definition would even allow to extend most of your results to arbitrary locally finite multigraphs (no matter whether connected or disconnected).

According to your suggestion we define the score of a vertex and the vertex-edge coloring number in such way that the two concepts are well-defined also for infinite graphs: The score of a vertex $v$ at a certain state $t$ of the game, $\operatorname{score}_{t}(v)$, is the number of marked edges incident with $v$ if $v$ is unmarked at state $t$, and 0 otherwise. The score of $v \in V(G)$ is $\operatorname{score}(v)=\sup _{t}\left\{\operatorname{score}_{t}(v)\right\}$, and the vertex-edge coloring number is $\operatorname{col}_{v e}(G)=\sup _{v \in V(G)}\{\operatorname{score}(v)\}+1$.

- (Comment 2) I wonder whether with the more natural definition (see Comment 1) the precondition "locally finite" is needed. There seems to be no need for it. Otherwise, please mention why and where you need the precondition "locally finite" to make the game well- defined.

Indeed. We could safely remove this condition.

- (Comment 3) In all theorems, lemmas, propositions and corollaries, please make clear in the formulation of the assertion and in the proofs whether they hold in general (i.e. for infinite multigraphs) or only for finite multigraphs.

It is now clearly indicated if a graph in a statement is finite and/or simple. After the definition of the vertex-edge coloring number, we explain that the invariant is well defined for both finite and infinite graphs and all results given in the paper hold for infinite graphs as well, unless otherwise is stated. We restrict to finite graphs just in two graph classes, planar and outerplanar graphs. For those two graph families we have not found the results about bounded degree orientation for the infinite case in the literature. In addition, the results about complete (multi)graphs clearly only hold in the finite case. 
In particular, Lemma 2 and its proofs should be formulated for the general case, since in Theorem 12 and Propositions 13 and 14 it has to be used for infinite graphs.

Indeed Lemma 3 (Lemma 2 in the previous version) is now stated and proved for the general case that can be used also for lattices.

Furthermore,

- the equation $p(G)=\left\lceil d^{*}(G)\right\rceil$ is only valid for finite multigraphs, since although the pseudoarboricity is well-defined for infinite multigraphs, your definition of maximum density does not apply to infinite multigraphs;

As mentioned and explained in the answer to Reviewer \#1, we have removed several concepts (including pseudoarbority) from various reasons, which makes this issue obsolete.

- the proof of Corollary 7 uses the maximum density, therefore Corollary 7 holds has been proven only for finite multigraphs and it may not be used in the proofs of Theorem 12, Propositions 13 and 14.

Propositions 11 and 12 (previous 13 and 14) are now proved by using Lemma 3 (Orientation Lemma), which has been proved also for infinite graphs.

- (Comment 4) In the last subcase of the second case in the proof of Proposition 10 and three times in the proof of Theorem 12, you use the fact that if in a graph there is a path of length $\geq 3$ whose inner vertices have degree 3 and are unmarked and whose outer edges are marked whereas its inner edges are unmarked (you have called such a path "free path" somewhere) and it is Bob's turn, then Bob has a strategy to force a score of at least 3. You might formulate this fact as a "Free-Path"-Lemma and prove it once and shorten the proofs of Proposition 10 and Theorem 12 by deleting the repeated argument and replacing it by a reference to the new lemma.

Done. Thank you for this nice suggestion!

- I suggest to explain where you use the preconditions $M(S)>s_{1}$ and $M\left(S^{\prime}\right)>s_{1}^{\prime}$ inn the proof of Lemma 15 (iv).

This issue was noticed also by Reviewer \#1. The fact is that condition $M(S)>s_{1}$ is not needed, hence we removed it. We also added a reason why $M\left(S^{\prime}\right)>s_{1}^{\prime}$ is needed. Indeed, if $M\left(S^{\prime}\right)=s_{1}^{\prime}$, then it is possible that $M(S)<M\left(S^{\prime}\right)$.

- I suggest to explain why property (iv) can be applied to $Q_{t}$ and $P_{t}$ (i.e., why does $M\left(Q_{t}\right)>2$ hold also in this case?)

The condition $M\left(Q_{t}\right)>m\left(Q_{t}\right)$ is not the precondition of $(i v)$ any more (see the previous comment), so we do not need to check if this holds.

- In the proof of Proposition 20 it is essential that Alice has the first move. Therefore the characterisation of graphs with vertex-edge coloring number at most 2 given in Proposition 20 holds only for connected graphs. It might be interesting to discuss the case of disconnected graphs as well. 
The proposition has been modified so that it gives the characterization of all (connected and disconnected) graphs $G$ with $\operatorname{col}_{v e}(G)=2$.

- (Comment 5) You might remark that the combination of Proposition 22 with Theorem 11 improves the general upper bound 5 for the game coloring number of cactuses given by Sidorowicz [1] to the value 3 in the special case of "subdivided" cactuses.

Done, thanks.

- It should be "coloring number of a CONNECTED graph $G$ yield the same ". You might also discuss disconnected graphs.

Since the characterization is now given for all graphs, CONNECTED is not needed any more.

- You might give a motivation for Question 5 (which might include a definition of the lexicographic graph product).

Done.

\section{Minor comments}

We have also checked all other (many) minor comments of the reviewer, and incorporated them to the revised version, with thanks. 


\title{
On a vertex-edge marking game on graphs
}

\author{
Boštjan Brešar ${ }^{a, b} \quad$ Nicolas Gastineau $^{c} \quad$ Tanja Gologranc ${ }^{a, b}$ \\ Olivier Togni ${ }^{c}$
}

April 7, 2020

${ }^{a}$ Faculty of Natural Sciences and Mathematics, University of Maribor, Slovenia

bostjan.bresar@um.si

tanja.gologranc1@um.si

${ }^{b}$ Institute of Mathematics, Physics and Mechanics, Ljubljana, Slovenia

${ }^{c}$ Laboratoire LIB, Université de Bourgogne Franche-Comté, France

Nicolas.Gastineau@u-bourgogne.fr

olivier.togni@u-bourgogne.fr

\begin{abstract}
The study of a variation of the marking game, in which the first player marks vertices and the second player marks edges of an undirected graph was proposed by Bartnicki et al. in [Game chromatic number of Cartesian product graphs, Electron. J. Combin. 15 (2008) \#R72]. In this game, the goal of the second player is to mark as many edges around an unmarked vertex as possible, while the first player wants just the opposite. In this paper, we prove various bounds for the corresponding graph invariant, the vertex-edge coloring number $\operatorname{col}_{v e}(G)$ of a graph $G$. In particular, every (finite or infinite) graph $G$ whose edges can be oriented in such a way that the maximum out-degree is bounded by an integer $d$ has $\operatorname{col}_{v e}(G) \leq d+2$. We investigate this invariant in (classes of) planar graphs, including some infinite lattices. We present a close connection between the vertex-edge coloring number of a graph $G$ and the game coloring number of the subdivision graph $S(G)$. In our main result, we bound the vertex-edge coloring number in complete graphs from below and from above, and while $\operatorname{col}_{v e}\left(K_{n}\right) \leq\left\lceil\log _{2} n\right\rceil+2$, the difference between the upper and the lower bound is roughly $\log _{2}\left(\log _{2} n\right)$. The latter results are in fact true for any multigraph whose underlying graph is $K_{n}$.
\end{abstract}

Keywords: marking game, coloring game, degenerate graph, complete graph

AMS Subj. Class. (2010): 05C15, 05C57

\section{Introduction}

The coloring game on graphs was introduced independently by Gardner [11] and Bodlander [4], and was henceforth studied by a number of authors. The initial version of the 
coloring game triggered numerous investigations, which resulted in the development of various methods and strategies; see the brief survey on different kinds of coloring games by Bartnicki et al. [3]. A close variation of the coloring game, which has been one of the main tools for bounding the game chromatic number, is the marking game as introduced by Zhu [17] (see also [1, 5, 12, 13, 14, 16, 18] for some further studies). The marking game can be viewed as the game version of the coloring number, which was introduced by Erdős and Hajnal [9] for infinite graphs. One can make a small modification of the definition from [17] so that it works for infinite graphs. The marking game is played on a graph $G$ by two players, Alice and Bob, who alternate turns in choosing a previously unchosen vertex $v$ of $G$; at the point $v$ is chosen its score $s(v)$ is determined as the cardinality of the set of (previously) chosen neighbors of $v$. The resulting invariant, the game coloring number is defined as $\operatorname{col}_{g}(G)=1+\sup \{s(x) \mid x \in V(G)\}$, where it is assumed that Alice's goal is to minimize and Bob's goal is to maximize the final score and both players play optimally. For all concepts mentioned, but not defined in this paper we refer to [7].

The vertex-edge marking game has been defined by Bartnicki et al. [2] as a variation of the marking game on vertices: as usual, two players play the game, and while the first player Alice marks vertices, Bob marks edges. The goal of Bob is to surround an unmarked vertex by as many marked edges as possible, while the goal of Alice is opposite; she wishes to keep the number of marked edges incident to an unmarked vertex as small as possible. The score of a vertex $v$ at a certain state $t$ of the game, $\operatorname{score}_{t}(v)$, is the number of marked edges incident with $v$ if $v$ is unmarked at state $t$, and 0 otherwise. The score of $v \in V(G)$ is score $(v)=\sup _{t}\left\{\right.$ score $\left._{t}(v)\right\}$, and the vertex-edge coloring number is

$$
\operatorname{col}_{v e}(G)=\sup _{v \in V(G)}\{\operatorname{score}(v)\}+1 .
$$

Note that the above definition of the vertex-edge coloring number is well defined for both finite and infinite graphs and all results we give hold for infinite graphs as well, unless otherwise stated. Also note that the vertex-edge marking game can be extended to multigraphs (allowing multiple edges between two vertices). We begin with the following obvious observation.

Lemma 1. For every multigraph $G$, if $H$ is a submultigraph of $G$, then we have $\operatorname{col}_{v e}(H) \leq$ $\operatorname{colve}_{v e}(G)$.

The vertex-edge coloring number of a graph $G$ is closely related to the game coloring number of the subdivision $S(G)$ of $G$, obtained by subdividing every edge of $G$ exactly once: 
Proposition 2. If $\operatorname{col}_{v e}(G)>2$, then $\operatorname{col}_{v e}(G)=\operatorname{col}_{g}(S(G))$.

(The proof of this result is given in Section 5.) Through this connection, one can derive from [13, Example 6.1] that $\operatorname{col}_{v e}\left(K_{n, n}\right)$ is unbounded when $n$ grows. Consequently, also $\left\{\operatorname{col}_{v e}\left(K_{n}\right) \mid n \in \mathbb{N}\right\}$ is unbounded, and we give some light on the asymptotic behaviour of the vertex-edge coloring number in complete graphs. More precisely, we prove that

$$
\left\lfloor\log _{2}(n-1)\right\rfloor-\left\lceil\log _{2}\left\lfloor\log _{2}(n-1)\right\rfloor\right\rceil+2 \leq \operatorname{col}_{v e}\left(K_{n}\right) \leq\left\lceil\log _{2} n\right\rceil+2 .
$$

In fact, the upper bound in (1) holds even if $K_{n}$ is generalized to $K_{n}^{(p)}$, where each edge of $K_{n}$ is replaced by the set of multiple edges of an arbitrarily large cardinality $p$. For the upper bound we model the game as a process on sorted words of integers that represent the positions in the game.

The paper is organized as follows. In the next section, we prove a basic auxiliary result, which we call the Orientation Lemma, and give several immediate consequences of this result, related to degeneracy and arboricity. In Section 3, we infer from the Orientation Lemma that $\operatorname{col}_{v e}(G) \leq 5$ in finite planar graphs $G$, and prove sharp upper bounds for the vertex-edge coloring number in cactus graphs and finite outerplanar graphs (which are 3 and 4, respectively). We also determine the exact value of the invariant in the hexagonal lattice and the square lattice, and bound it in the triangular lattice. Section 4 is concerned with complete graphs and the proof of (1). In Section 5 we prove Proposition 2, and in the last section we propose several open problems.

\section{Constrained degree orientations}

In this section, we present a general upper bound for the vertex-edge coloring number of a graph which involves orientations of its edges. We also give some immediate consequences of this result that will be applied in several proofs of subsequent sections.

A graph has a $d$-bounded orientation if its edges can be oriented in such a way that the maximum out-degree of the vertices in the resulting digraph is at most $d$. The concept was introduced under this name by Chrobak and Eppstein [6] although similar concepts have been studied earlier (see e.g. [10]).

Lemma 3 (Orientation Lemma). If $G$ is a graph which has a d-bounded orientation, then colve $(G) \leq d+2$.

Proof. Consider an oriented digraph $D$ obtained from $G$ by orienting its edges in such a way that $\operatorname{outdeg}(v) \leq d$ for all $v \in V(G)$. Alice's strategy to maintain $\operatorname{score}(v) \leq d+1$ for every vertex $v$ is as follows. Suppose that Bob marked an edge $e=u v$, and $u \rightarrow v$ is the orientation of $e$ in $D$. Then Alice marks $v$ if $v$ has not yet been marked (and 
otherwise she marks any unmarked vertex). Note that at the point $v$ was marked, $u v$ is the only marked edge in $G$ for which the orientation is towards $v$. Since $v$ has outdegree at most $d$, there are thus at most $d+1$ edges incident to $v$ that were already marked at the time $v$ is marked. Hence score $(v) \leq d+1$, which gives the claimed statement.

Another well-known parameter related to bounded-degree orientations is degeneracy. A $k$-degenerate graph is a graph in which every subgraph has a vertex incident with at most $k$ edges. Alternatively, a graph is $k$-degenerate if and only if there is an ordering of its vertices such that every vertex $v$ has at most $k$ backward edges with respect to the ordering. Note that a $k$-degenerate graph has a $k$-bounded orientation. Bounded orientation is also in relation with arboricity: if $G$ can be decomposed into $k$ forests, then it has a $k$-bounded orientation (simply orient each tree towards a root). Hence, by Lemma 3, we have the following bound.

Corollary 4. Given a positive integer $k$, if $G$ is a $k$-degenerate graph or if its edge-set can be decomposed into $k$ forests, then colve $(G) \leq k+2$.

Since $\operatorname{col}_{v e}\left(P_{6}\right)=3$ we get the following result.

Corollary 5. For any forest $F$ we have $\operatorname{col}_{v e}(F) \leq 3$ and the bound is tight.

\section{Planar graphs}

Using the tools of Section 2 and other known results, we derive sharp upper bounds for the vertex-edge coloring number in several classes of planar graphs. In particular, we find exact values of the vertex-edge coloring numbers of two infinite lattices.

First, since every finite planar graph has a 3-bounded orientation (which can even be constructed in linear time, see [6]), we infer by the Orientation Lemma the following general bound for planar graphs.

Proposition 6. For every finite planar graph we have colve $(G) \leq 5$.

We next present an auxiliary result concerning a lower bound for the vertex-edge coloring number, for which we need the following definition. In a graph $G$ on which the vertex-edge marking game is played, a free-path is a path $P$ of length at least 3 whose inner vertices have degree at least 3 in $G$ and are unmarked and whose endvertices have incident marked edges in $P$.

Lemma 7 (Free-path Lemma). If at some state in the vertex-edge coloring game, a graph $G$ contains a free-path and it is Bob's turn, then Bob has a strategy to force $\operatorname{col}_{v e}(G) \geq 4$. 
Proof. Let $P=\left(x_{1}, x_{2}, \ldots, x_{\ell+1}\right)$ be a free-path of length $\ell \geq 3$ in $G$. We prove the lemma by induction on $\ell$. If $\ell=3$ then Bob marks the edge $x_{2} x_{3}$ (if it is already marked, then he marks an arbitrary unmarked edge), leading to two unmarked vertices $x_{2}$ and $x_{3}$ with score 2. Thus, whatever the choice of Alice, there will remain an unmarked vertex of score 2 and Bob will mark an edge incident with it to obtain a score of 3. Assume now the lemma is true for free-paths of length at most $\ell^{\prime}=\ell-1$. We prove that Bob can ensure a score of 3 with the free-path $P$ of length $\ell$. Bob marks the edge $x_{2} x_{3}$ (or an arbitrary unmarked edge if $x_{2} x_{3}$ is already marked) and then either Alice marks $x_{2}$ and then we have a free-path $\left(x_{2}, x_{3}, \ldots, x_{\ell+1}\right)$ of length $\ell-1$ which, by induction, gives the result or else, in the case Alice does not mark $x_{2}$, Bob can mark an unmarked edge incident with $x_{2}$ (if $x_{2}$ has all its incident edges marked, then it has already a score of at least 3 ) to obtain a score of 3 on $x_{2}$, which implies $\operatorname{col}_{v e}(G) \geq 4$.

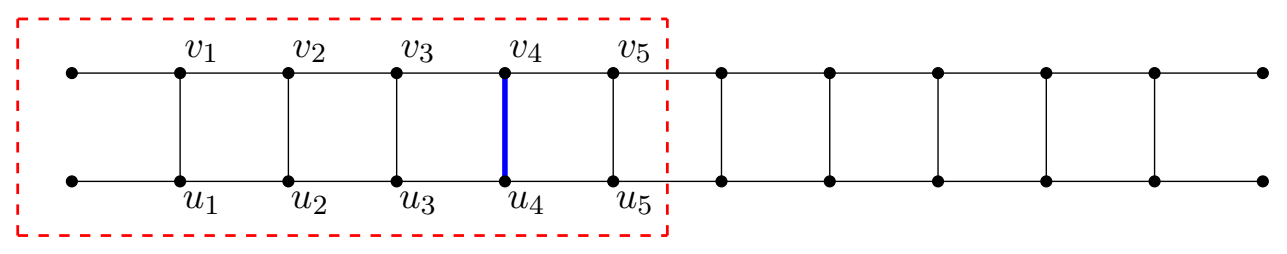

Figure 1: The graph $H$ and one of the two disjoint copies of the graph $G$ (inside the dashed area) contained in $H$; the thick line indicating the first edge chosen by Bob.

We are now able to prove the following result for outerplanar graphs. The Cartesian product $G \square H$ of two graphs $G$ and $H$ is the graph with vertex set $V(G) \times V(H)$ and in which vertices $\left(g_{1}, h_{1}\right)$ and $\left(g_{2}, h_{2}\right)$ are adjacent if either $g_{1} g_{2} \in E(G)$ and $h_{1}=h_{2}$, or $g_{1}=g_{2}$ and $h_{1} h_{2} \in E(H)$.

Proposition 8. Let $G$ be a finite outerplanar graph, then $\operatorname{col}_{v e}(G) \leq 4$ and the bound is tight.

Proof. Outerplanar graphs are 2-degenerate, hence Corollary 4 gives the upper bound. For the tightness, we prove that the graph $H=P_{10} \square P_{2}$ with a leaf added on each of its four vertices of degree 2 satisfies $\operatorname{col}_{v e}(H) \geq 4$. Let $G$ be the graph $P_{5} \square P_{2}$ with an added leaf on two adjacent vertices of degree 2 . We let $u_{i}$ and $v_{i}$, for $i \in\{1, \ldots, 5\}$ be the vertices of $G$ such that, for $i \in\{1,2,3,4\}, u_{i}, v_{i}, u_{i+1}$ and $v_{i+1}$ is an induced square of $G\left(u_{i} v_{i}, u_{i+1} v_{i+1}, u_{i} u_{i+1}\right.$ and $v_{i} v_{i+1}$ being the edges of this square). Remark that there are two disjoint copies of $G$ in $H$ (see Figure 1). Consequently, whatever the first vertex marked by Alice, there remains one copy of $G$ unmarked by Alice. The strategy of Bob starts by marking the edge $u_{4} v_{4}$ of this unmarked copy of $G$ in $H$ (see Figure 1). 
First, if Alice does not mark $u_{4}$ or $v_{4}$, then there is at least one edge consisting of unmarked vertices among $u_{3} v_{3}$ and $u_{5} v_{5}$ and Bob marks this edge, say it is $u_{3} v_{3}$. Whatever the vertex Alice marks next, there is a free-path of length 3 between either $v_{3}$ and $v_{4}$ or between $u_{3}$ and $u_{4}$, and hence by Lemma 7 there is a vertex of score 3 .

Second, if Alice marks $u_{4}$ or $v_{4}$, then suppose, without loss of generality, that Alice has marked $v_{4}$. In this case Bob marks the edge $u_{2} v_{2}$. We distinguish two cases. In the case Alice does not mark $u_{2}$ or $v_{2}$, then Bob marks an edge containing only non-marked vertices among $u_{1} v_{1}$ and $u_{3} v_{3}$ and whatever the vertex Alice marks, Bob can mark an edge between two unmarked vertices so that they are now both incident with two marked edges. In the case Alice marks $u_{2}$, there is a free-path $\left(v_{4}, u_{4}, u_{3}, v_{3}, v_{2}, u_{2}\right)$ and thus by Lemma 7, Bob can force a score of at least 3 in some vertex. Otherwise if Alice marks $v_{2}$, then there is again a free-path $\left(v_{4}, u_{4}, u_{3}, u_{2}, v_{2}\right)$, hence, again by Lemma 7 , $\operatorname{col}_{v e}(G) \geq 4$.

A cactus graph is a connected graph in which any two simple cycles have at most one vertex in common. Such graphs have a tree structure, i.e., each of its blocks is either a cycle or an edge and the intersection graph of its blocks is a tree. Since every cactus graph $G$ is outerplanar, we have $\operatorname{col}_{v e}(G) \leq 4$ by Proposition 8 for such graphs. However, we prove a stronger bound in the next theorem.

Theorem 9. For every cactus graph $G_{C}$, we have colve $\left(G_{C}\right) \leq 3$.

Proof. Assume that $G_{C}$ has at least one cycle $C_{1}$ since otherwise $G_{C}$ would be a tree and hence Proposition 6 would allow to conclude.

Then in each other block $B$ of $G_{C}$, there is a unique vertex $x$ that is closer to $C_{1}$ than the other vertices of $B$ (by the tree structure of the cactus). We call this vertex $x$ the head of $B$. For the cycle $C_{1}$, we choose an arbitrary vertex to be the head.

The strategy of Alice is the following:

R1. At the beginning, Alice marks any vertex of $C_{1}$.

R2. If Bob has marked an edge $e$ that does not lie in a cycle, then if possible, Alice marks the head of $e$, otherwise (if the head is already marked) Alice marks an arbitrary unmarked vertex of $G_{C}$.

R3. If Bob has marked an edge of a cycle $C$ of $G_{C}$ and no other edges of $C$ are marked, then if possible, Alice marks the head of $C$, otherwise she marks an arbitrary unmarked vertex of $G_{C}$.

R4. Otherwise, if Bob marks an edge $e=u v$ of $C$ and $C$ had already marked edges, then if possible, Alice marks among $u$ and $v$ the vertex that is closer to the first marked 
edge of $C$ along the path that does not cross the head of $C$. If this is not possible (the chosen vertex is already marked), then Alice marks an arbitrary vertex of $G_{C}$.

We now prove that with this strategy for Alice, there will not exist an unmarked vertex $u$ that is incident with more than two marked edges. If $u$ is not in a cycle, then by Rule R2, only the edge emanating from $u$ towards the root cycle $C_{1}$ and at most one edge in the other direction may be marked at the time $u$ is marked. If $u$ lies in a cycle $C$ of $G_{C}$ and $u$ has both of its two incident edges in $C$ marked, then other edges incident with $u$ are not marked, by Rules R2 and R3. In addition, by Rule R4, Alice will mark $u$ when both of the incident edges of $u$ in $C$ are marked. Otherwise, if at most one edge in $C$, which is incident with $u$, is marked, then by Rules $\mathrm{R} 2$ and $\mathrm{R} 3, u$ will be marked as the head of any other edge (or the head of the corresponding cycle) with which $u$ is incident. Hence, in either case, as soon as two edges incident with $u$ are being marked, $u$ will be marked, thus the score of $u$ is at most 2 .

Theorem 10. If $\mathcal{H}$ is the infinite hexagonal lattice, then $\operatorname{col}_{\text {ve }}(\mathcal{H})=4$.

Proof. It is easy to orient the edges of $\mathcal{H}$ in such a way that each vertex has out-degree at most 2 (see Figure 2 showing a portion of the hexagonal lattice with the orientation of edges depicted), hence, by Lemma 3 , we infer $\operatorname{col}_{v e}(\mathcal{H}) \leq 4$. To prove the lower bound, we are going to show that Bob has a strategy which ensures a score of 3 in some vertex of $\mathcal{H}$.

Consider a sufficiently large portion of the hexagonal lattice such that after Alice's first move, Bob is able to mark an edge $e_{1}=x_{1} y_{1}$ that is far enough from the vertex marked by Alice (distance 7 should suffice). See Figure 2 for the names of the other edges and vertices considered. If Alice does not mark $x_{1}$ or $y_{1}$, then Bob marks an edge $e \in\left\{e_{11}, e_{12}\right\}$ such that all vertices of the 6-cycle $C$ containing $e_{1}$ and $e$ are unmarked. Hence whatever the vertex Alice marks there is a free-path of length 4 on $C$ and by Lemma 7, Bob has a way to force a score of 3 .

Now assume, without loss of generality, that Alice has marked vertex $x_{1}$. Then Bob marks the edge $e_{2}=x_{2} y_{2}$. Suppose that in the next move Alice does not mark vertex $y_{1}$. Then there remains a free path between either $x_{2}$ (if $y_{2}$ is not marked) or $y_{2}$ (if $x_{2}$ is not marked) and $x_{1}$ and by Lemma 7, Bob has a way to force a score of 3 in some vertex.

Otherwise, if Alice marks $y_{1}$ then Bob marks the edge $e_{3}=x_{3} y_{3}$, by which he gets two marked edges of the 6 -cycle, none of which vertices is marked. Hence, whatever the vertex marked by Alice, there will remain a free-path between $x_{2}$ or $y_{2}$ and $x_{3}$ or $y_{3}$ and thus Bob will be able again to force a score of 3 , yielding $\operatorname{col}_{v e}(\mathcal{H}) \geq 4$.

Proposition 11. If $\mathcal{S}$ is the infinite square lattice, then $\operatorname{colve}_{v e}(\mathcal{S})=4$. 


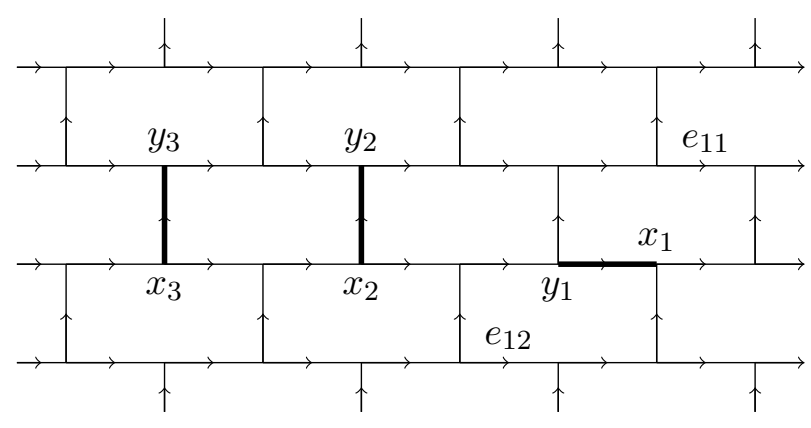

Figure 2: A part of the hexagonal lattice with 2-bounded orientation and some designated edges and vertices.
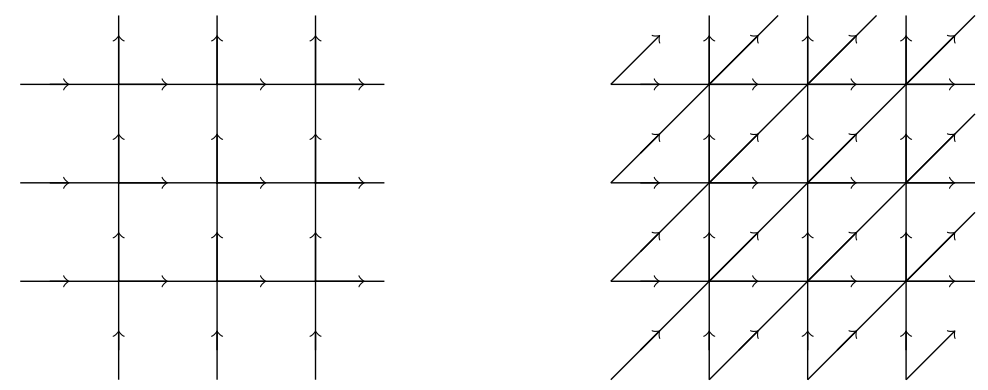

Figure 3: The square lattice with 2-bounded orientation and the triangular lattice with 3-bounded orientation.

Proof. Since the graph $H$ from the proof of Proposition 8 is a subgraph of $\mathcal{S}$, it follows from Lemma 1 that $4=\operatorname{col}_{v e}(H) \leq \operatorname{col}_{v e}(\mathcal{S})$.

For the upper bound we use the Orientation Lemma, noting that $\mathcal{S}$ can be oriented in such a way that the out-degree of every vertex of $\mathcal{S}$ is bounded by 2 , see Figure 3.

For the triangular lattice, there is an orientation of its edges such that the out-degree of every vertex is 3 (see Figure 3), hence we infer by Lemma 3 the following upper bound.

Proposition 12. If $\mathcal{T}$ is the infinite triangular lattice, then $\operatorname{col}_{v e}(\mathcal{T}) \leq 5$.

Since $\mathcal{H}$ is a spanning subgraph of $\mathcal{T}$, we infer the lower bound $\operatorname{col}_{v e}(\mathcal{T}) \geq 4$. We wonder what is the exact value of the vertex-edge coloring number of $\mathcal{T}$. The question is related also to the exact upper bound of this number in planar graphs.

\section{Complete graphs}

In order to prove the upper bound on the vertex-edge marking game on complete graphs, we find convenient to model the game as a process on sorted words of integers that will 
represent the positions of the game (i.e., the number of incident marked edges of each unmarked vertex). This model enables us to prove the upper bound for a family of multigraphs that generalize complete graphs; notably, given a positive integer $n$ and a non-zero cardinal number $p$, the multigraph $K_{n}^{(p)}$ has $n$ vertices and between each pair of vertices there are $p$ parallel edges.

We first introduce some notation and two lemmas on sorted words. Let $S$ be a finite sequence of non-negative integers in non-increasing order, i.e., let $S=s_{1} s_{2} \cdots s_{p}$ be a word over the alphabet of integers, with $s_{1} \geq s_{2} \geq \cdots \geq s_{p} ; p=|S|$. This is called a sorted word. We consider the process that starts from a sorted word $S$ and apply inductively the operation that consists in suppressing the first letter $s_{1}$ of the word and adding the value 1 to two distinct letters of the word (and then reordering the letters of the word in such a way that it becomes a sorted word). More formally, let $f$ be a function that maps a sorted word $S=s_{1} \cdots s_{p}$, where $p \geq 3$, to a sorted word $T=t_{1} \cdots t_{p-1}$, such that there exist $i, j \in\{2, \ldots, p\}, i \neq j$, and $s_{k}^{\prime}=s_{k+1}$ for $j \neq k+1 \neq i$, and $s_{i-1}^{\prime}=s_{i}+1, s_{j-1}^{\prime}=s_{j}+1$, and $T$ is obtained from $S^{\prime}=s_{1}^{\prime} s_{2}^{\prime} \cdots s_{p-1}^{\prime}$ by an eventual reordering of $S^{\prime}$ to create a non-increasing order. We then write $f(S)=T$. When $p=2$, we let $f\left(s_{1} s_{2}\right)=s_{2}+1$ (that is, the resulting word $T$ is of length 1 ).

Let $M(S)$ be the maximum integer in a word that can be obtained by this process starting from the word $S$. More formally, let $m(S)$ be the maximum (i.e., the first) integer of a word $S$. Then let $\mathcal{S}_{S}=\left\{S^{\prime} \mid \exists f_{1}, \ldots, f_{k}: S^{\prime}=f_{k} \circ \ldots \circ f_{1}(S)\right\}$. Alternatively, we can define $M(S)$ as $\max \left\{m\left(S^{\prime}\right) \mid S^{\prime} \in \mathcal{S}_{S}\right\}$.

We also define the partial order $\preceq$ on sorted words by $S \preceq S^{\prime}$ if $|S| \leq\left|S^{\prime}\right|$ and for each $i, 1 \leq i \leq|S|, s_{i} \leq s_{i}^{\prime}$.

Lemma 13. The following properties hold for sorted words.

(i) if $S \preceq S^{\prime}$, then $M(S) \leq M\left(S^{\prime}\right)$;

(ii) if $f(S)=S^{\prime}$, then $M\left(S^{\prime}\right) \leq M(S)$;

(iii) for any positive integer $i, M\left(1^{i}\right)=1+M\left(0^{i}\right)$;

(iv) if $M\left(S^{\prime}\right)>s_{1}^{\prime},|S|=\left|S^{\prime}\right|$, and $s_{i} \geq s_{i}^{\prime}$ for every integer $i, i \geq 2$, then $M(S) \geq$ $M\left(S^{\prime}\right)$.

(v) for any integers $r \geq 2$ and $s \geq 2$, we have $M\left(1^{r} 0^{s}\right) \leq M\left(1^{r+1} 0^{s-2}\right)$.

Proof. (i) Let $S_{m}$ be the word of $\mathcal{S}_{S}$ such that $m\left(S_{m}\right)=M(S)$. Let $f_{1}, \ldots, f_{k}$ be the functions such that $S_{m}=f_{k} \circ \ldots \circ f_{1}(S)$. Since $S \preceq S^{\prime}$, then clearly, for the word $S_{m}^{\prime}=f_{k} \circ \ldots \circ f_{1}\left(S^{\prime}\right)$ we have $m\left(S_{m}^{\prime}\right) \geq m\left(S_{m}\right)=M(S)$.

(ii) If $f(S)=S^{\prime}$, then $\mathcal{S}_{S^{\prime}} \subseteq \mathcal{S}_{S}$, which implies that $M\left(S^{\prime}\right) \leq M(S)$. 
(iii) Let $S_{m}$ be a sorted word of $\mathcal{S}_{0^{i}}$ such that $m\left(S_{m}\right)=M\left(0^{i}\right)$. The same sequence of functions used to obtain $S_{m}$ from $0^{i}$ can be used on $1^{i}$ to obtain a sorted word $S_{m}^{\prime}$ such that $m\left(S_{m}^{\prime}\right)=M\left(1^{i}\right)$. We infer $m\left(S_{m}^{\prime}\right)=m\left(S_{m}\right)+1$.

(iv) Let $S_{m}^{\prime}$ be the word of $\mathcal{S}_{S^{\prime}}$ such that $m\left(S_{m}^{\prime}\right)=M\left(S^{\prime}\right)$. Since $M\left(S^{\prime}\right)>s_{1}^{\prime}$, there exist the set of functions $f_{1}, \ldots, f_{k}$ such that $S_{m}^{\prime}=f_{k} \circ \ldots \circ f_{1}\left(S^{\prime}\right)$. Note that $f_{k} \circ \ldots \circ f_{1}(S)$ yields a sorted word $S_{m}$ such that $M(S) \geq m\left(S_{m}\right) \geq m\left(S_{m}^{\prime}\right)=M\left(S^{\prime}\right)$.

(v) Let us prove that for any integers $r \geq 2$ and $s \geq 2$, we have $M\left(1^{r} 0^{s}\right) \leq M\left(1^{r+1} 0^{s-2}\right)$. Let $1^{r} 0^{s}=P_{0}, P_{1}, \ldots, P_{n}=S_{m}$ be a sequence of words with $f_{i}\left(P_{i}\right)=P_{i+1}$ for $0 \leq i \leq$ $n-1$, and $M\left(1^{r} 0^{s}\right)=m\left(S_{m}\right)$. Let $t$ be the smallest integer such that $P_{t}$ does not contain the subword $0^{s}$. (If such an integer $t$ does not exists, then we have $M\left(1^{r} 0^{s}\right)=M\left(1^{r}\right) \leq$ $M\left(1^{r+1} 0^{s-2}\right)$, by property (i), as desired.) Let $Q_{1}=1^{r+1} 0^{s-2}$. For any $i, 2 \leq i \leq t$, we denote by $Q_{i}$ the sorted word $f_{i-2} \circ \cdots f_{0}\left(Q_{1}\right)$. See the diagram on Figure 4 .

$$
\begin{gathered}
P_{0}=1^{r} 0^{s} \longrightarrow f_{0} P_{1}=f_{0}\left(1^{r} 0^{s}\right)-\underset{f_{1} \ldots f_{t-2}}{\longrightarrow} P_{t-1}=f_{t-2} \circ \cdots \circ f_{0}\left(1^{r} 0^{s}\right) \\
Q_{1}=1^{r+1} 0^{s-2} \underset{f_{0}}{\longrightarrow} Q_{2}=f_{0}\left(1^{r+1} 0^{s-2}\right) \underset{f_{1} \ldots f_{t-2}}{\stackrel{-->}{f_{t-2}}} Q_{t}=f_{t-2} \circ \cdots \circ f_{0}\left(1^{r+1} 0^{s-2}\right)
\end{gathered}
$$

Figure 4: Illustration of the proof of Lemma 13.

Note that $P_{t-1}=f_{t-2} \circ \cdots \circ f_{0}\left(1^{r} 0^{s}\right)=u_{1} \ldots u_{r-t+1} 0^{s}$, and $Q_{t}=f_{t-2} \circ \cdots \circ$ $f_{0}\left(1^{r+1} 0^{s-2}\right)=u_{1} \ldots u_{r-t+1} 1^{1} 0^{s-2}$ (since $f_{1}, \ldots f_{t-2}$ are functions whose composition changes the $r$ first integers equal to 1 into $\left.u_{1} \ldots u_{r-t+1}\right)$. Observe that $P_{t}$ is obtained from $P_{t-1}$ by deleting the first integer and adding 1 to two integers, at least one of which is 0 .

We distinguish two cases. If $P_{t}$ was obtained from $P_{t-1}$ by adding 1 to two integers equal to 0 , then $P_{t}=u_{2} \ldots u_{r-t+1} 1^{2} 0^{s-2}$, and clearly $P_{t} \preceq Q_{t}$, which implies $M\left(P_{t}\right) \leq$ $M\left(Q_{t}\right)$ by property (i). Thus, we have $M\left(1^{r} 0^{s}\right)=M\left(P_{t}\right) \leq M\left(Q_{t}\right) \leq M\left(1^{r+1} 0^{s-2}\right)$.

The second case is that $P_{t}$ is obtained from $P_{t-1}$ by suppressing $u_{1}$, changing one integer 0 to 1 and increasing by 1 an integer $u_{i}$, where $i \in\{2, \ldots, r-t+1\}$. Suppose $u_{i}<u_{2}$. Then, let $j$ be the largest index in $\{2, \ldots, i-1\}$ such that $u_{j}>u_{i}$. Hence,

$$
P_{t}=u_{2} \ldots u_{j}\left(u_{i}+1\right) u_{j+1} \ldots u_{i-1} u_{i+1} \ldots u_{r-t+1} 1^{1} 0^{s-1} .
$$

Note that $P_{t} \preceq Q_{t}$, since $u_{j} \geq u_{i}+1$. We again derive in the same way that $M\left(1^{r} 0^{s}\right) \leq$ $M\left(1^{r+1} 0^{s-2}\right)$. Finally, suppose that $u_{i}=u_{2}$. Then $P_{t}=\left(u_{2}+1\right) u_{3} \ldots u_{r-t+1} 10^{s-1}$. If $M\left(P_{0}\right)=M\left(P_{t}\right)=u_{2}+1$, then $M\left(Q_{t}\right) \geq u_{2}+1$, because for $Q^{\prime}=f\left(Q_{t}\right)$, where $f$ is a function that increases the second integer by 1 , we get $M\left(Q_{t}\right) \geq M\left(Q^{\prime}\right) \geq u_{2}+1$. 
Otherwise, if $M\left(P_{t}\right)>u_{2}+1$, then by property (iv) applied on $Q_{t}$ and $P_{t}$, we get $M\left(Q_{t}\right) \geq$ $M\left(P_{t}\right)$. In either case, we infer $M\left(1^{r} 0^{s}\right)=M\left(P_{t}\right) \leq M\left(Q_{t}\right) \leq M\left(1^{r+1} 0^{s-2}\right)$.

The properties of Lemma 13 are used to prove the following lemma.

Lemma 14. For any $k \geq 1$ we have $M\left(0^{2^{k}+1}\right)=k+1=\left\lceil\log _{2}\left(2^{k}+1\right)\right\rceil$.

Proof. Let $t=2^{k}+1$, where $k \geq 1$. Using the sequence of functions that always change two zeros, we get the sequence of words, $0^{t}, 1^{2} 0^{t-3}, \ldots, 1^{\left\lceil\frac{t}{2}\right\rceil-1} 0^{2}, 1^{\left\lceil\frac{t}{2}\right\rceil}$. Therefore $M\left(1^{\left\lceil\frac{t}{2}\right\rceil}\right) \leq M\left(0^{t}\right)$.

Now, we show the reversed inequality, $M\left(0^{t}\right) \leq M\left(1^{\left\lceil\frac{t}{2}\right\rceil}\right)$. Since $\mathcal{S}_{0^{t}}=\mathcal{S}_{1^{2} 0^{t-3}} \cup\left\{0^{t}\right\}$, we have $M\left(0^{t}\right)=M\left(1^{2} 0^{t-3}\right)$. If $k=1$, that is, $t=3$, this gives $M\left(0^{t}\right)=M\left(0^{3}\right)=$ $M\left(1^{2}\right)=M\left(1^{\left\lceil\frac{t}{2}\right\rceil}\right)$. Otherwise, we apply Lemma $13(\mathrm{v})$ several times and we get $M\left(0^{t}\right)=$ $M\left(1^{2} 0^{t-3}\right) \leq M\left(1^{3} 0^{t-5}\right) \leq \ldots \leq M\left(1^{\left\lceil\frac{t}{2}\right\rceil}\right)$.

We prove $M\left(0^{2^{k}+1}\right)=k+1$ by induction on $k$. When $k=1$, we clearly have $M(000)=2$. By the above, $M\left(0^{2^{k}+1}\right)=M\left(1^{2^{k-1}+1}\right)$. By Lemma 13(iii), this is in turn equal to $1+M\left(0^{2^{k-1}+1}\right)$, which is by induction equal to $k$. Hence, $M\left(0^{2^{k}+1}\right)=k+1$.

Corollary 15. For any $n \geq 3$, we have $M\left(0^{n}\right) \leq\left\lceil\log _{2} n\right\rceil+1$.

Proof. For $n \geq 3$, let $k$ be the integer such that $2^{k-1}+1<n \leq 2^{k}+1$. Thus, by Lemma 13(i) and Lemma $14, M\left(0^{n}\right) \leq M\left(0^{2^{k}+1}\right)=k+1 \leq\left\lceil\log _{2} n\right\rceil+1$.

We think that in fact $M\left(0^{n}\right)=\left\lceil\log _{2} n\right\rceil$, but could not improve the upper bound from Corollary 15. We are now ready to prove the upper bound for $\operatorname{col}_{v e}\left(K_{n}^{(p)}\right)$.

Theorem 16. For a non-zero cardinal number $p$ and every $n \geq 2$,

$$
\operatorname{col}_{v e}\left(K_{n}^{(p)}\right) \leq\left\lceil\log _{2} n\right\rceil+2 .
$$

Proof. We are going to prove the upper bound $\left\lceil\log _{2} n\right\rceil+2$ for the multigraph $K_{n}^{(p)}$, where $p \geq n-1$. Then, Lemma 1 yields the statement of the theorem for any $p<n-1$ as well.

The strategy of Alice is to mark at each step a vertex having a maximum number of incident edges that are marked. We will prove that whatever the strategy of Bob, there will be no unmarked vertex with more than $\left\lceil\log _{2} n\right\rceil+1$ incident marked edges. Clearly, at any step for which there remain at least two unmarked vertices, we can assume that Bob marks an edge $e=x y$ with both $x$ and $y$ being not already marked, by which the score of two vertices is increased. When just one vertex $x$ remains unmarked by Alice, then Bob marks an edge incident with $x$ increasing its score by 1 (therefore, before the penultimate move of Alice, $x$ and $y$ have been unmarked, and if they have the same 
number of incident marked edges at that time, then it is possible that $\operatorname{col}_{v e}\left(K_{n}^{(p)}\right)$ is attained only by the score of $x$ ). With this hypothesis, we can represent the game by a sequence $S_{0}, S_{1}, \ldots, S_{n-1}$ of sorted words of integers as described above; word $S_{i}$, where $0 \leq i \leq n-1$, is the sorted word that contains the numbers of marked edges incident with each unmarked vertex of $K_{n}^{(p)}$ after the $i$ th move of Bob. In addition, $S_{i}$ is obtained from $S_{i-1}$ by a function as described above. Since we have $S_{0}=0^{n}$, then, by virtue of Corollary 15, we obtain $M\left(S_{0}\right) \leq\left\lceil\log _{2} n\right\rceil+1$, and hence $\operatorname{col}_{v e}\left(K_{n}^{(p)}\right) \leq\left\lceil\log _{2} n\right\rceil+2$.

Clearly, plugging $p=1$ in Theorem 16 , we get $\operatorname{col}_{v e}\left(K_{n}\right) \leq\left\lceil\log _{2} n\right\rceil+2$. Note that Lemma 14 implies that $\operatorname{col}_{v e}\left(K_{n}\right) \leq\left\lceil\log _{2} n\right\rceil+1$, for $n=2^{k}+1$.

Now, we prove the lower bound in (1) by presenting a strategy for Bob for which at least one vertex will have a score of $\left\lfloor\log _{2}(n-1)\right\rfloor-\left\lceil\log _{2}\left\lfloor\log _{2}(n-1)\right\rfloor\right\rceil+1$ whatever Alice's strategy.

Theorem 17. For every $n \geq 3$, we have

$$
\operatorname{col}_{v e}\left(K_{n}\right) \geq\left\lfloor\log _{2}(n-1)\right\rfloor-\left\lceil\log _{2}\left\lfloor\log _{2}(n-1)\right\rfloor\right\rceil+2 .
$$

Proof. First, we consider the graph $K_{n}$, where $n=2^{k}+1$. The strategy of Bob consists of several steps, in each of which Bob marks edges of a matching. After the $i$ th step Bob can ensure that there exists a subgraph $G_{i}$ with $2^{k-i}$ unmarked vertices each of which is incident with $i$ marked edges.

Note that Alice starts the game by marking an arbitrary vertex $x$. Let $X_{0}=\{x\}$ and $G_{0}=K_{n}-X_{0}$. Clearly, $G_{0}$ has $2^{k}$ unmarked vertices each of which is incident with 0 marked edges (which presents the zero-th step).

We follow with the first step and it is Bob's turn. In the next $2^{k-1}$ moves Bob marks edges of a perfect matching of $G_{0}$. During this time, Alice marks $2^{k-1}$ vertices (denote this set of vertices by $X_{1}$ ) of $G_{0}$. Let $G_{1}=G_{0}-X_{1}$, and note that $G_{1}$ has (at least) $2^{k-1}$ unmarked vertices each of which is incident with 1 marked edge. This ends the first step and note that Alice was the last to play in this step.

In the $i$ th step we note by induction that there exists a subgraph $G_{i-1}$ with $2^{k-i+1}$ unmarked vertices each of which is incident with $i-1$ marked edges. If there exists a perfect matching in $G_{i-1}$ that consists of non-marked edges, then in the next $2^{k-i}$ moves Bob marks edges of this perfect matching. During this time, Alice marks $2^{k-i}$ vertices (denote this set of vertices by $X_{i}$ ) of $G_{i-1}$. Then $G_{i}=G_{i-1}-X_{i}$ has (at least) $2^{k-i}$ unmarked vertices each of which is incident with $i$ marked edges. We apply Dirac's theorem [8], which ensures a Hamiltonian cycle in a graph $H$ with even order if each vertex has degree at least half of the order. This in turn implies the existence of a 
perfect matching in $H$. Therefore, Bob can ensure the existence of a perfect matching of non-marked edges in $G_{i-1}$ if

$$
\left|V\left(G_{i-1}\right)\right|-1-(i-1) \geq \frac{1}{2}\left|V\left(G_{i-1}\right)\right| .
$$

That is, $2^{k-i+1}-i \geq 2^{k-i}$, which gives

$$
2^{k-i} \geq i
$$

The number of steps (in Bob's strategy) is the largest $i$ such that (2) is fulfilled. When this condition is no longer fulfilled (after the $i$ th step), Bob can mark an edge incident to an unmarked vertex of $G_{i}$ by which the score of this vertex is at least $i+1$ (and so $\left.\operatorname{col}_{v e}\left(K_{n}\right) \geq i+2\right)$.

Since $i \in \mathbb{N}$, the largest $i$ satisfying (2) is $k-\left\lceil\log _{2} k\right\rceil$ or $k-\left\lceil\log _{2} k\right\rceil+1$ (depending on $k)$. In the case $n=2^{k}+1$, we get $\operatorname{col}_{v e}\left(K_{n}\right) \geq \log _{2}(n-1)-\left\lceil\log _{2}\left(\log _{2}(n-1)\right)\right\rceil+2$.

Finally, let $2^{k} \leq n-1<2^{k+1}$. Therefore,

$$
\operatorname{col}_{v e}\left(K_{n}\right) \geq \operatorname{col}_{v e}\left(K_{2^{k}+1}\right) \geq k-\left\lceil\log _{2} k\right\rceil+2 \geq\left\lfloor\log _{2}(n-1)\right\rfloor-\left\lceil\log _{2}\left\lfloor\log _{2}(n-1)\right\rfloor\right\rceil+2 .
$$

\section{Relations with the marking game}

We will prove that the vertex-edge coloring number of a graph $G$ coincides with the game coloring number of the graph $S(G)$ obtained from $G$ by subdividing all of its edges once, as soon as the vertex-edge coloring number of $G$ is at least 3 (Proposition 2).

First, we prove that the class of graphs $G$ with $\operatorname{col}_{v e}(G) \leq 2$ is small. Clearly, only graphs with no edges have this number equal to 1 . We characterize the graphs with $\operatorname{col}_{v e}(G)=2$ as follows.

Proposition 18. If $G$ is a non-empty graph, then $\operatorname{col}_{v e}(G)=2$ if and only if $G$ is a forest with at most one connected component of diameter at most 4 and all other connected components of diameter at most 2.

Proof. First, suppose that $\operatorname{col}_{v e}(G)=2$. Remark that if Bob can mark an edge having each of its end-vertices unmarked and incident to an unmarked edge, then it implies $\operatorname{col}_{v e}(G) \geq 3$. If $G$ contains a cycle, such an edge can be found in Bob's first move. Thus $G$ is a forest. If $G$ has a connected component $T$ of diameter at least 5 or if $G$ contains 
two connected components both having diameter at least 3, such an edge can be found at first Bob's move whatever the vertex Alice has chosen in her first move.

For the converse, let $G$ be a non-empty forest with at most one connected component $T_{1}$ of diameter at most 4 and all other connected components $T_{2}, \ldots, T_{k}$ of diameter at most 2. Let $c_{i}$ be a center of $T_{i}$ for any $i \in\{1, \ldots, k\}$. The strategy of Alice is to first mark $c_{1}$ and then after each Bob's move (in which he marks an edge $e$ ), she marks (if possible) an unmarked vertex incident with $e$ that is not a leaf. Therefore the score of each vertex in $G$ is at most 1 and $\operatorname{col}_{v e}(G)=2$.

For the purpose of proving the next result, which connects the vertex-edge marking game on a graph $G$ with the (standard) marking game on the subdivided graph $S(G)$, we propose two variations of the vertex-edge marking game. In the first variation, which we call vertex-edge-star-Alice marking game, Alice is allowed to play also on the edges while Bob's role does not change. The corresponding score of the game will be denoted by $\operatorname{col}_{v e}^{* A}(G)$, and is defined exactly the same as in the standard vertex-edge marking game, that is, $\sup _{v \in V(G)}\{\operatorname{score}(v)\}+1$. As in the vertex-edge marking game, $\operatorname{score}(v)=$ $\sup _{t}\left\{\operatorname{score}_{t}(v)\right\}$, where score $t$ is the number of marked edges surrounding the vertex $v$ at state $t$ if $v$ is unmarked, and 0 if $v$ is marked at state $t$. Since Alice may choose to play on the vertices of $G$ as long as possible also in the vertex-edge-star-Alice marking game, and it is not to her advantage to play on the edges, it is clear that the new invariant gives the same score.

Similarly, we call vertex-edge-star-Bob marking game the game in which Bob is allowed to play also on the vertices while Alice's role does not change. The corresponding score of the game will be denoted by $\operatorname{col}_{v e}^{* B}(G)$, and is again defined in the same way as above. Since Bob may choose to play on the edges of $G$ as long as possible in this version of the vertex-edge marking game, and it is not to his advantage to play on the vertices, we get the following observation.

Lemma 19. For any graph $G, \operatorname{col}_{v e}^{* A}(G)=\operatorname{col}_{v e}(G)=\operatorname{col}_{v e}^{* B}(G)$.

We are now able to prove Proposition 2.

Proof of Proposition 2. Consider a strategy of Alice played in the vertex-edge-star-Bob marking game on $G$, which bounds score $(v)$ from above by $\operatorname{col}_{v e}^{* B}(G)-1$ for all vertices $v$ of $G$. Alice can use the same strategy in the marking game in the graph $S(G)$ by playing only on the original vertices of $G$. During the marking game on $S(G)$, she will imagine a vertex-edge-star-Bob marking game be played on $G$, and will copy her moves from the optimal strategy on the vertex-edge-star-Bob marking game on $G$ to the real game played on $S(G)$. Note that the resulting score $s(v)$ of a vertex $v$ is bounded by $\operatorname{col}_{v e}^{* B}(G)-1$ if 
Alice plays optimally (since the score of subdivided vertices is at most 3, the maximum score will be achieved by an original vertex except possibly when $\operatorname{col}_{v e}(G)=3$ ). This gives $\operatorname{col}_{g}(S(G)) \leq \operatorname{col}_{v e}^{* B}(G)$.

To see the reversed inequality, let us consider a strategy of Bob in the vertex-edgestar-Alice marking game, which ensures that $\sup _{v \in V(G)}\{\operatorname{score}(v)\}$ of a vertex $v$ in $G$ is at least $\operatorname{col}_{v e}^{* A}(G)-1$. While playing the marking game on $S(G)$, Bob uses this strategy in the vertex-edge-star-Alice marking game on $G$, by playing the subdivided vertices of $S(G)$ in the corresponding order. In this way, $\sup \{s(v) \mid v \in V(G)\} \geq \operatorname{col}_{v e}^{* A}(G)-1$, which gives $\operatorname{col}_{g}(S(G)) \geq \operatorname{col}_{v e}^{* A}(G)$. By Lemma 19, the proof follows.

The above result implies that the results in this paper for the vertex edge coloring number of a graph $G$ yield the same results for the game coloring number of the subdivision graph $S(G)$, as soon as $G$ is not a forest with at most one connected component of diameter at most 4 and all other connected components of diameter at most 2. (Otherwise, one can check that, for instance, $\operatorname{col}_{v e}\left(P_{3}\right)=2$, yet $\operatorname{col}_{g}\left(S\left(P_{3}\right)\right)=\operatorname{col}_{g}\left(P_{5}\right)=3$.) Combining Proposition 2 with Theorem 9 we can improve the general upper bound 5 for the game coloring number of cactus graphs given by Sidorowicz [15] to the value 3 in the special case of subdivided cactus graphs.

\section{Concluding remarks}

There are a number of well studied classes of graphs for which it would be interesting to establish whether the vertex-edge coloring number is bounded by a constant. (Clearly, if a class of graphs is $k$-degenerate for some fixed $k$, then Corollary 4 provides a positive answer.) In particular, we propose to consider the class of hypercubes, and pose the following

Question 1. Is $\left\{\operatorname{col}_{v e}\left(Q_{n}\right) \mid n \in \mathbb{N}\right\}$, where $Q_{n}$ denotes the hypercube of dimension $n$, bounded by a constant?

As proven in Section 3, finite planar graphs admit a general upper bound of 5 for their vertex-edge coloring number. There are several examples of (finite or infinite) planar graphs $G$ with $\operatorname{col}_{v e}(G)=4$, so we wonder what is the correct sharp bound in planar graphs. We thus pose the following question.

Question 2. Is there a (finite) planar graph $G$ with $\operatorname{col}_{v e}(G)=5$ ?

(Note that for infinite planar graphs we did not establish a general upper bound for the vertex-edge marking game.) It seems that a good candidate for which Question 2 could have an affirmative answer is the triangular lattice. 
Question 3. Is $\operatorname{col}_{v e}(\mathcal{T})$ for the triangular lattice $\mathcal{T}$ equal to 4 or 5 ?

Trivially, the following general upper bound $\operatorname{col}_{v e}(G) \leq \Delta(G)+1$ holds in every graph $G$. Note that $\operatorname{col}_{v e}\left(C_{n}\right)=3$ for any $n \geq 3$, hence the bound is attained in cycles, as well as in the hexagonal lattice, $\operatorname{since} \operatorname{col}_{v e}(\mathcal{H})=4$. We propose the problem of characterizing the graphs $G$ in which $\operatorname{col}_{v e}(G)=\Delta(G)+1$, and pose the question about the most interesting case.

Question 4. For which graphs $G$ with maximum degree 3 we have colve $_{\text {ve }}(G)=4$ ?

A logarithmic upper bound for complete graphs, see Theorem 16, suggests that in many classes of finite graphs the vertex-edge coloring number is bounded by a constant. Therefore, it would be interesting to find a graph operation by which one could built a family of finite graphs with unbounded vertex-edge coloring number. We think that the lexicographic product of graphs could be such an operation. Let $G$ and $H$ be finite graphs. The lexicographic product $G \circ H$ of $G$ and $H$ has $V(G \circ H)=V(G) \times V(H)$, and $(g, h)\left(g^{\prime}, h^{\prime}\right) \in E(G \circ H)$ if either $g=g^{\prime}$ and $h h \in E(H)$, or $g g^{\prime} \in E(G)$. We propose the following question for which we suspect it has an affirmative answer.

Question 5. Is it true that $\operatorname{col}_{v e}\left(G \circ K_{4}\right) \geq \operatorname{col}_{v e}(G)+1$ ? More generally, is colve $(G \circ$ $\left.K_{2^{n+1}}\right) \geq \operatorname{col}_{v e}(G)+n$ ?

\section{Acknowledgement}

We are grateful to both anonymous referees for their valuable suggestions.

This work was performed with the financial support of the bilateral project "Distanceconstrained and game colorings of graph products" (BI-FR/18-19-Proteus-011).

B.B. and T.G. acknowledge the financial support from the Slovenian Research Agency (research core funding No. P1-0297 and project Contemporary invariants in graphs No. J1-9109).

\section{References}

[1] S.D. Andres, A. Theuser, Note on the game colouring number of powers of graphs, Discuss. Math. Graph Theory 36 (2016) 31-42.

[2] T. Bartnicki, B. Brešar, J. Grytczuk, M. Kovše, Z. Miechowicz, I. Peterin, Game chromatic number of Cartesian product graphs, Electron. J. Combin. 15 (2008) \#R72, 13 pp. 
[3] T. Bartnicki, J. Grytczuk, H. A. Kierstead, X. Zhu, The map coloring game, Amer. Math. Monthly 14 (2007) 793-803.

[4] H.L. Bodlaender, On the complexity of some coloring games, Internat. J. Found. Comput. Sci. 2 (1991) 133-147.

[5] C. Charpentier, S. Dantas, C. de Figueiredo, A. Furtado, S. Gravier, On NordhausGaddum type inequalities for the game chromatic and game coloring numbers, Discrete Math. 342 (2019) 1318-1324.

[6] M. Chrobak, D. Eppstein, Planar orientations with low out-degree and compaction of adjacency matrices, Theoret. Comput. Sci. 86 (1991) 243-266.

[7] R. Diestel, Graph Theory, fourth ed., in: Graduate Texts in Mathematics, vol. 173, Springer, Heidelberg, 2010.

[8] G.A. Dirac, Some theorems on abstract graphs, Proc. Lond. Math. Soc. 2 (1952) 69-81.

[9] P. Erdős, A. Hajnal, On chromatic number of graphs and set-systems, Acta Math. Acad. Sci. Hungar 17 (1966) 61-99.

[10] A. Frank, A. Gyárfás, How to orient the edges of a graph ?, Combinatorics (Proc. Fifth Hungarian Colloq., Keszthely, 1976), Vol. I, (1978) 353-364, North-Holland, Amsterdam-New York, 1978.

[11] M. Gardner, Mathematical games, Scientific American 244 (1981) 18-26.

[12] H. Kierstead, B. Mohar, S. Špacapan, D. Yang, X. Zhu, The two-coloring number and degenerate colorings of planar graphs, SIAM J. Discrete Math. 23 (2009) 15481560.

[13] H.A. Kierstead, T. Trotter, Competitive colorings of oriented graphs, Electron. J. Combin. 8 (2001) \#R12, 15pp.

[14] H.A. Kierstead, D. Yang, Very asymmetric marking games, Order 22 (2005) 93-107.

[15] E. Sidorowicz, The game chromatic number and the game colouring number of cactuses, Inform. Process. Lett. 102 (2007) 147-151.

[16] D. Yang, X. Zhu, Activation strategy for asymmetric marking games, European J. Combin. 29 (2008) 1123-1132. 
[17] X. Zhu, The game coloring number of planar graphs, J. Combin. Theory Ser. B 75 (1999) 245-258.

[18] X. Zhu, Refined activation strategy for the marking game, J. Combin. Theory ser. B 98 (2008) 1-18. 